\title{
The Atmospheric Scanning Electron Microscope (ASEM) observes the Cultured Fluorescent Neuron
}

\author{
Tatsuhiko Ebihara ${ }^{1}$, Hidetoshi Nishiyama ${ }^{2}$, Mitsuo Suga ${ }^{2}$ and Chikara Sato ${ }^{1}$, \\ 1. National Institute of Industrial Science and Technology (AIST), Tsukuba, 305-8566, Japan \\ 2. JEOL Ltd., 1-2 Musashino 3-chome, Akishima, Tokyo 196-8558, Japan
}

Correspondence should be addressed to Chikara Sato (ti-sato@aist.go.jp)

Neural network by neurite and synapse, is the bases of brain functions and development. The morphological changes of neurons and synapses are a lead to solution of the brain development and memory formation. Optical microscopic observation of cultured neurons, is important method for understanding brain. As synapses are around $1 \mu \mathrm{m}$, optical microscopic observation faces difficulties for subsynaptic structures. One solution is super-resolution optical microscopy, the other, we subject the possibility of electron microscopic observation.

The new Atmospheric Scanning Electron Microscope (ASEM) is Correlative Light-Electron Microscope (CLEM), in which the optical microscope (OM) and SEM quasi-simultaneously observe samples in water solution under open atmosphere [1]. In this system, an inverted SEM observes the underwater sample from beneath an open dish while an optical microscope (OM) observes it from above (Fig. 1). The disposable dish with an electron transparent SiN film window can hold a few milliliters of culture medium, and allows various types of cells to be cultured in a stable environment, e.g., a $\mathrm{CO}_{2}$ incubator. The film with poly-L-lysine coating allows primary neural culture of mouse hippocampus (Fig. 2). Since ASEM observes sample in water, samples need only brief pretreatment, but no treatment against vacuum is required. Immuno-staining is also as easy as those for normal OM [2]. ASEM would realize the easy EM observation and prevent the effects caused by the hydrophobic pretreatments for traditional EM.

We developed the heavy metal staining conditions to visualize cell shape and intracellular structures. Double staining by uranyl acetate and tannic acid, stained plasma membrane and cell nuclei. Tungstic acid stained mitochondria and other fibers. We imaged primary neural cultures whose postsynaptic spines are transgenetically labeled by green fluorescence. In combination with $\mathrm{OM}$ and heavy metal staining (EM), fine structures of neurites could be distinguished in pre- and post- synaptic structures (Fig. 2). We also immunostained cultured neurons, and studied the distribution of structural proteins in growth cone. 


\section{References}

[1] H. Nishiyama et al., J Struct Biol 169, (2010) p. 438-449.

[2] Y. Maruyama et al., J Struct Biol 180, (2012) p. 259-270.

\section{Atmospheric SEM (ASEM)}

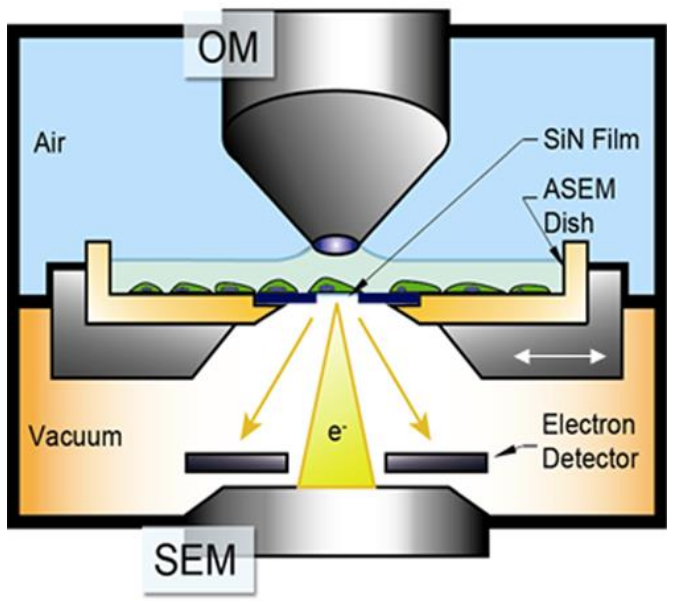

Figure 1. Configuration of the ASEM and cultured, fixed and stained cells on the ASEM dish. The SEM has a totally inverted structure, with electron gun at the bottom.

\section{Div.14 Cultured Neuron}
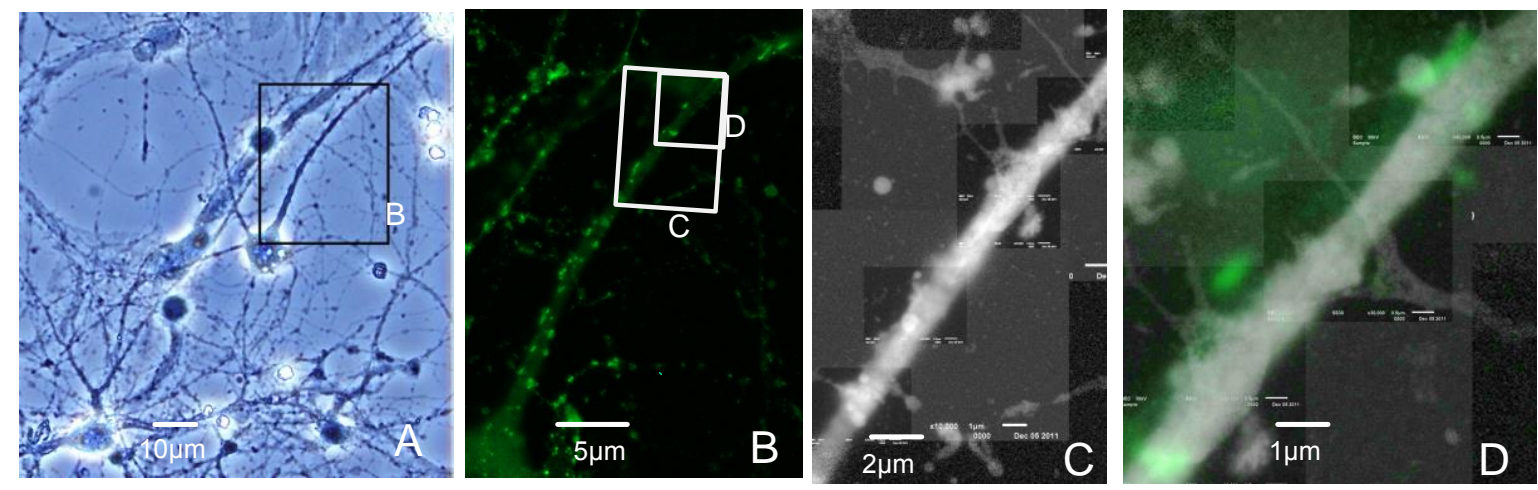

Figure 2. Correlative imaging of cultured neuron (Day in vitro 14). (A) Phase contrast (B) Fluorescent image of rectangle area in (A). (C) EM image. (D) Merged image of OM and EM, the same area in Fig B's rectangle. 